\title{
A transformation method to study the solvability of fully coupled FBSDEs
}

\author{
Stefan Ankirchner \\ Alexander Fromm* \\ Julian Wendt
}

October 6, 2020

\begin{abstract}
We consider fully coupled forward-backward stochastic differential equations (FBSDEs), where all function parameters are Lipschitz continuous, the terminal condition is monotone and the diffusion coefficient of the forward part depends monotonically on $z$, the control process component of the backward part. We show that there exists a class of linear transformations turning the FBSDE into an auxiliary FBSDE for which the Lipschitz constant of the forward diffusion coefficient w.r.t. $z$ is smaller than the inverse of the Lipschitz constant of the terminal condition w.r.t. the forward component $x$. The latter condition allows to verify existence of a global solution by analyzing the spatial derivative of the decoupling field. This is useful since by applying the inverse linear transformation to a solution of the auxiliary FBSDE we obtain a solution to the original one. We illustrate with several examples how linear transformations, combined with an analysis of the decoupling field's gradient, can be used for proving global solvability of FBSDEs.
\end{abstract}

\section{Introduction}

Let $T>0$ and let $W=\left(W_{t}\right)_{t \in[0, T]}$ be a Brownian motion defined on a complete filtered probability space $\left(\Omega, \mathcal{F},\left(\mathcal{F}_{t}\right)_{t \in[0, T]}, \mathbb{P}\right)$, where the filtration is defined by $\mathcal{F}_{t}:=$ $\sigma\left(\mathcal{N},\left(W_{s}\right)_{s \in[0, t]}\right)$ with $\mathcal{N}$ denoting the set of all $\mathbb{P}$-null sets.

In this article we introduce a new method for checking whether the forwardbackward stochastic differential equation (FBSDE)

$$
\begin{aligned}
X_{t} & =x+\int_{0}^{t} \mu\left(r, X_{r}, Y_{r}, Z_{r}\right) d r+\int_{0}^{t} \sigma\left(r, X_{r}, Y_{r}, Z_{r}\right) d W_{r}, \\
Y_{t} & =\xi\left(X_{T}\right)-\int_{t}^{T} f\left(r, X_{r}, Y_{r}, Z_{r}\right) d r-\int_{t}^{T} Z_{r} d W_{r}, t \in[0, T],
\end{aligned}
$$

possesses a solution, where we make the following assumptions on the parameter functions $\mu, \sigma, \xi$ and $f$ :

${ }^{*}$ Financial support from the German Research Foundation through the project AN 1024/4-1 is gratefully acknowledged 
(A1) $\mu, \sigma, f: \Omega \times[0, T] \times \mathbb{R} \times \mathbb{R} \times \mathbb{R} \rightarrow \mathbb{R}$ are progressively measurable, meaning that for all $t \in[0, T]$ the functions $\mu, \sigma, f$ restricted to $\Omega \times[0, t] \times \mathbb{R} \times \mathbb{R} \times \mathbb{R}$ are $\mathcal{F}_{t} \otimes \mathcal{B}([0, t]) \otimes \mathcal{B}(\mathbb{R}) \otimes \mathcal{B}(\mathbb{R}) \otimes \mathcal{B}(\mathbb{R})$-measurable,

(A2) $\mu, \sigma, f$ are Lipschitz continuous in $(x, y, z)$ with Lipschitz constant $L$, i.e. for all $t \in[0, T]$ and almost all $\omega \in \Omega$ we have

$$
\left|\varphi\left(t, x_{1}, y_{1}, z_{1}\right)-\varphi\left(t, x_{2}, y_{2}, z_{2}\right)\right| \leq L\left(\left|x_{1}-x_{2}\right|+\left|y_{1}-y_{2}\right|+\left|z_{1}-z_{2}\right|\right),
$$

for all $x_{i}, y_{i}, z_{i} \in \mathbb{R}, i=1,2$ and $\varphi=\mu, \sigma, f$.

(A3) $\|\mu(\cdot, \cdot, 0,0,0)\|_{\infty}+\|\sigma(\cdot, \cdot, 0,0,0)\|_{\infty}+\|f(\cdot, \cdot, 0,0,0)\|_{\infty}<\infty$,

(A4) $\xi: \Omega \times \mathbb{R} \rightarrow \mathbb{R}$ is measurable, Lipschitz continuous in the second variable and satisfies $\|\xi(\cdot, 0)\|_{\infty}<\infty$.

(A5) $\xi$ is monotonically increasing in the second variable, while $\sigma$ is monotonically decreasing in $z$.

Notice that (A1)-(A4) are standard assumptions on the measurability, Lipschitz continuity and growth of the parameters. Observe that the FBSDE (1.1) is fully coupled, that is, the dynamics of the forward part depend on the solution of the backward component and, vice versa, the dynamics of the backward part depend on the forward component. Recall that for fully coupled FBSDEs the assumptions (A1)-(A4) are not sufficient for the existence of a solution (see Example 1.1 below for a counterexample). We obtain a sufficient condition for the existence of a solution, on a sufficiently small time interval, by adding the assumption

$$
L_{\xi, x}<L_{\sigma, z}^{-1}
$$

where $L_{\xi, x}$ denotes the smallest Lipschitz constant of $\xi$ w.r.t. the second component $x$; and $L_{\sigma, z}$ denotes the smallest Lipschitz constant of $\sigma$ w.r.t. the last component $z$. By $L_{\sigma, z}^{-1}=\frac{1}{L_{\sigma, z}}$ we mean $\frac{1}{L_{\sigma, z}}$ if $L_{\sigma, z}>0$ and $\infty$ otherwise. We interpret $L_{\xi, x}^{-1}$ in the same way.

We refer to condition (1.2) as the contraction condition, as it guarantees that one can define, via a Picard iteration, a process sequence $\left(X^{n}, Y^{n}, Z^{n}\right)$ that is contracting with respect to some nice norm, defined on a sufficiently small time interval. The limit of the Picard sequence can be shown to converge to a solution of (1.1) on the small time interval considered. Thus, the contraction condition (1.2), together with (A1)-(A4), is sufficient for the existence of a local solution of (1.1) (see Theorem 3.4 below for a precise statement). In the following we refer to the union of the five conditions (A1)-(A4) and (1.2) as the standard Lipschitz condition (SLC).

A method for checking global solvability for FBSDEs satisfying SLC is to verify that the so-called decoupling field (see Section 3 for the definition) is Lipschitz continuous with a Lipschitz constant bounded away from $L_{\sigma, z}^{-1}$, uniformly on the maximal existence interval. Whether the Lipschitz constant is bounded away from $L_{\sigma, z}^{-1}$ can be checked by studying the dynamics of difference quotients or the differential quotients of the solution processes $(X, Y, Z)$ with respect to the initial condition of the forward equation. The first approach using difference quotients is pursued in 
[11], with the aim to provide a collection of various sufficient conditions for global solvability. The second approach is followed in [7] (see also [8]) in order to describe a recipe for checking global sovability of FBSDEs. The recipe is based on checking whether the absolute value of the decoupling field's gradient with respect to $x$ stays bounded away from $L_{\sigma, z}^{-1}$, and in the following we refer to it as the method of decoupling fields.

If (1.2) is not satisfied, then one can try to apply a linear transformation the process pair $(X, Y)$ in order to arrive at an auxiliary FBSDE satisfying the contraction condition. In this article we show that this is indeed always possible under the monotonicity condition (A5). We provide a class of linear transformations leading to an FBSDE satisfying the contraction condition and we prove that if the new FBSDE has a solution, then so does the original FBSDE (1.1). Indeed, from a solution of the auxiliary FBSDE we construct a solution of (1.1). Thus, for checking whether the FBSDE (1.1) has a solution it is enough to study the auxiliary FBSDE. Since the latter satisfies SLC, the method of decoupling fields applies to it.

The monotonicity of $\sigma$ in $z$ allows to choose the transformation in such a way that the control process $Z$ is uniquely determined by the control process of the auxiliary FBSDE. Concerning (A5) we want to point out the following:

- If $\sigma$ is monotonically increasing while $\xi$ is monotonically decreasing one can define $(\hat{X}, \hat{Y}, \hat{Z}):=(-X, Y, Z)$ to obtain a new FBSDE with diffusion coefficient $\hat{\sigma}:=-\sigma$ and terminal condition $\hat{\xi}:=\xi(-\cdot)$ that satisfies (A5). Note that this transformation is invertible, this means that if one FBSDE has a solution on some time interval so does the other.

- If $\sigma$ and $\xi$ are both monotonically increasing or decreasing in $z$ and $x$, respectively, then it can happen that even a local solution does not exist (see Example 1.1 below). Therefore, in this case an invertible linear transformation leading to an FBSDE with (1.2) does not necessarily exist.

The idea to use linear transformations when studying the solvability of FBSDEs can be found already in the literature. Theorems 6.2 and 6.3 in [11] provide sufficient conditions for (local) solvability, and the proofs are based on linear transformations leading to FBSDEs possessing solutions. The assumptions on the parameters of the FBSDEs, however, do not cover our setting (A1)-(A5). Moreover, the structure of the transformations used in [11] differs from the transformations we consider in this paper.

In the article [1] a linear transformation has been used in order to reduce a specific FBSDE to an auxiliary FBSDE for which a global solution is shown to exist. Indeed, the example of [1] raised the question of whether there exists a general class of FBSDEs for which a linear transformation is helpful in studying global solvability. The present article gives an affirmative answer.

As pointed out above, the condition (1.2) allows to check global solvability of fully coupled FBSDEs by studying the Lipschitz continuity of the decoupling field. There are several other approaches for proving global solvability that do not primarily target the Lipschitz continuity of the decoupling field. In [10] and [5] the link between FBSDEs and PDEs is exploited in order to prove global solvability of FBSDEs, where the diffusion coefficient $\sigma$ does not depend on $z$ and satisfies a non-degeneracy 
condition. This method is usually referred to as the Four Step Scheme. Notice that we do not assume that $\sigma$ is non-degenerate. More importantly, our method is developed particularly for the case where $\sigma$ depends on $z$.

There are several probabilistic approaches for proving solvability under some monotonicity conditions on the function parameters. Pardoux and Tang [14] make a monotonicity assumption on $\mu$ with respect to $x$ and $f$ with respect to $y$ and show existence via contraction arguments. Another approach is the so-called Method of Continuation, presented e.g. in [15], [17] and [9]. In these articles the existence of a global solution to an FBSDE is provided by imposing a kind of monotonicity on the parameter functions with respect to all variables $x, y$ and $z$. In contrast, in the present article we impose monotonicity of $\sigma$ in $z$ and of $\xi$ in $x$. Note, however, that our conditions do not guarantee global existence, but they allow to apply the method of decoupling fields to verify this.

We finally mention the monograph [12] and the article [11] for a more detailed overview on existing approaches.

The paper is organized as follows: In section 2, we derive a class of transformations leading to FBSDEs satisfying SLC. In section 3, we introduce decoupling fields and present a local existence result for FBSDEs satisfying SLC. In section 4, we present our main results. We give a complete account of the conditions needed for the linear transformations, and of the conditions leading to solvability of the original FBSDE. Finally, in section 5 we illustrate with several explicit examples how linear transformations and the method of decoupling fields can be used for analyzing the solvability of fully coupled FBSDE satisfying (A1)-(A5), but not (1.2).

We close the introduction with an example showing that if an FBSDE satisfies (A1)-(A4), but neither (A5) nor (1.2), then a solution needn't to exist, even on an arbitrarily small interval.

Example 1.1 (cf. Example 2.3.1 in [7]). Let $\sigma_{0} \in \mathbb{R} \backslash\{0\}$ be a constant. We consider for some $t \in[0, T)$ the FBSDE

$$
\begin{aligned}
& X_{s}=x+\int_{t}^{s}\left(\sigma_{0}+Z_{r}\right) d W_{r}, \\
& Y_{s}=X_{T}-\int_{s}^{T} Z_{r} d W_{r}, \quad s \in[t, T] .
\end{aligned}
$$

This means that

- $\mu$ and $f$ vanish,

- $\sigma$ is defined via $\sigma(s, x, y, z)=\sigma_{0}+z$,

- $\xi=\operatorname{Id}_{\mathbb{R}}$.

We now claim that equation (1.3) cannot have a progressively measurable solution, no matter how small $T-t>0$ is chosen.

In fact, the forward equation implies

$$
X_{T}-X_{s}=\int_{s}^{T}\left(\sigma_{0}+Z_{r}\right) d W_{r}=\sigma_{0}\left(W_{T}-W_{s}\right)+\int_{s}^{T} Z_{r} d W_{r}
$$


or

$$
X_{T}-\int_{s}^{T} Z_{r} d W_{r}=X_{s}+\sigma_{0}\left(W_{T}-W_{s}\right), \quad s \in[t, T] .
$$

Together with the backward equation we obtain

$$
Y_{s}=X_{s}+\sigma_{0}\left(W_{T}-W_{s}\right)
$$

which for $s=t$ means

$$
Y_{t}-x=\sigma_{0}\left(W_{T}-W_{t}\right) .
$$

This cannot be true, however, since $Y_{t}$ is $\mathcal{F}_{t}$-measurable and $\sigma_{0}\left(W_{T}-W_{t}\right)$ is a non-degenerate Gaussian random variable independent of $\mathcal{F}_{t}$.

\section{Transformations leading to SLC}

In this section we motivate the choice of transformations that we consider in this paper. We want to find linear transformations of the processes $X$ and $Y$ such that the transformed FBSDEs satisfy SLC. In other words, our goal is to determine $a, b, c, d \in \mathbb{R}$ such that the processes $\hat{X}, \hat{Y}, \hat{Z}$, given by

$$
\left(\begin{array}{c}
\hat{X}_{t} \\
\hat{Y}_{t}
\end{array}\right):=\left(\begin{array}{ll}
a & b \\
c & d
\end{array}\right)\left(\begin{array}{c}
X_{t} \\
Y_{t}
\end{array}\right)
$$

and $\hat{Z}_{t}:=c \sigma\left(t, X_{t}, Y_{t}, Z_{t}\right)+d Z_{t}$, "fulfill" a FBSDE with parameters that satisfy SLC. Our goal is then to examine the solvability of the new FBSDE and transfer the results to our original one. In order to make this work, we have to assume that the transformation is invertible, i.e. that we can recover $(X, Y, Z)$ from $(\hat{X}, \hat{Y}, \hat{Z})$. Thus, we have to choose $a, b, c, d$ such that $a d-b c \neq 0$ and the map $z \mapsto c \sigma(\omega, t, x, y, z)+d z$ is invertible for all $(\omega, t, x, y) \in \Omega \times[0, T] \times \mathbb{R} \times \mathbb{R}$.

Whether the choice of the parameters $a, b, c, d$ is suitable depends on the monotonicity of the functions $\sigma$ and $\xi$. To explain this dependence we introduce the constants $K_{\sigma, z}, K_{\xi, x} \geq 0$ as the largest constants satisfying

$$
\begin{aligned}
\left|\sigma\left(t, x, y, z_{1}\right)-\sigma\left(t, x, y, z_{2}\right)\right| & \geq K_{\sigma, z}\left|z_{1}-z_{2}\right|, \\
\left|\xi\left(x_{1}\right)-\xi\left(x_{2}\right)\right| & \geq K_{\xi, x}\left|x_{1}-x_{2}\right|,
\end{aligned}
$$

for all $x_{1}, x_{2}, y, z_{1}, z_{2} \in \mathbb{R}$ and $t \in[0, T]$. Note that such constants always exist since the above inequalities are satisfied by replacing $K_{\sigma, z}$ or $K_{\xi, x}$ with 0 . In case $K_{\sigma, z}>0$ or $K_{\xi, x}>0$ we have strict monotonicity for $\sigma$ or $\xi$ with slope of at least $K_{\sigma, z}$ or $K_{\xi, x}$, respectively.

For the sake of simplicity we restrict our considerations in this section to the case where $\xi(\omega, \cdot)$ and $\sigma(\omega, x, y, \cdot)$ are differentiable for all $\omega \in \Omega, x, y \in \mathbb{R}$, and $K_{\xi, x}=0$ holds true. However, we prove in the following section that the properties derived in this section also hold true in the more general setting of this paper requiring only Lipschitz continuity and $K_{\xi, x} \geq 0$. 
Note that the transformed FBSDE takes the form

$$
\begin{aligned}
& \hat{X}_{t}=x+\int_{0}^{t} \hat{\mu}\left(s, \hat{X}_{s}, \hat{Y}_{s}, \hat{Z}_{s}\right) d s+\int_{0}^{t} \hat{\sigma}\left(s, \hat{X}_{s}, \hat{Y}_{s}, \hat{Z}_{s}\right) d W_{s}, \\
& \hat{Y}_{t}=\hat{\xi}\left(\hat{X}_{T}\right)-\int_{t}^{T} \hat{f}\left(s, \hat{X}_{s}, \hat{Y}_{s}, \hat{Z}_{s}\right) d s-\int_{t}^{T} \hat{Z}_{s} d W_{s}, t \in[0, T],
\end{aligned}
$$

where the parameters are given by

$$
\begin{aligned}
\hat{\mu}(\omega, s, x, y, z) & :=a \mu(\omega, s, \Phi(s, x, y, z))+b f(\omega, s, \Phi(s, x, y, z)) \\
\hat{\sigma}(\omega, s, x, y, z) & :=a \sigma(\omega, s, \Phi(s, x, y, z))+b \hat{\varphi}(\omega, s, x, y, z) \\
\hat{f}(\omega, s, x, y, z) & :=c \mu(\omega, s, \Phi(s, x, y, z))+d f(\omega, s, \Phi(s, x, y, z)) \\
\hat{\xi}(\omega, x) & :=\left((c \operatorname{Id}+d \xi(\omega, \cdot)) \circ(a \operatorname{Id}+b \xi(\omega, \cdot))^{-1}\right)(x),
\end{aligned}
$$

and

$$
\begin{aligned}
\varphi(\omega, s, x, y, z) & :=(c \sigma(\omega, s, x, y, \cdot)+d \mathrm{Id})^{-1}(z), \\
\hat{\varphi}(\omega, s, x, y, z) & :=\varphi\left(\omega, s, \frac{d x-b y}{a d-b c}, \frac{-c x+a y}{a d-b c}, z\right), \\
\Phi(s, x, y, z) & :=\left(\frac{d x-b y}{a d-b c}, \frac{-c x+a y}{a d-b c}, \hat{\varphi}(s, x, y, z)\right),
\end{aligned}
$$

for $(\omega, s, x, y, z) \in \Omega \times[0, T] \times \mathbb{R} \times \mathbb{R} \times \mathbb{R}$. We emphasize that without loss of generality we can assume that $a, c \geq 0$, because one can just replace $(a, b)$ with $(-a,-b)$ if $a \leq 0$, and $(c, d)$ with $(-c,-d)$ if $c \leq 0$. That does not change the Lipschitz constants $L_{\hat{\sigma}, z}, L_{\hat{\xi}, x}$. Consequently, we consider only the case where $a, c \geq$ 0 . Moreover, we assume the following to ensure that the above definitions make sense and we can estimate the Lipschitz constants of $\hat{\xi}$ and $\hat{\sigma}$ by $L_{\hat{\xi}, x} \leq 1<L_{\hat{\sigma}, z}$ :

(i) $a>0$ and $b \geq 0$,

(ii) $d \leq 0$, in particular, $d<0$ if $K_{\sigma, z}=0$,

(iii) $c>0$ and $|d|$ small enough.

The first point ensures that the mapping $a \mathrm{Id}+b \xi: \mathbb{R} \rightarrow \mathbb{R}$ is a bijection and thus $\hat{\xi}$ is well-defined. The second point implies that the function $c \sigma(s, x, y, \cdot)+d \mathrm{Id}$ is invertible which is necessary for the definition of $\varphi$. The last point finally allows to derive a nice estimate for $L_{\hat{\xi}, x}$ in $(2.4)$ because then $0 \leq c+d \xi^{\prime}(x) \leq c$.

Now, since we have established that $a, c>0$ and $b \geq 0, d \leq 0$ should hold true, we claim that, without loss of generality, we can assume that $a, c=1$ (with adaptation of $b, d)$ as the following Lemma proves.

Lemma 2.1. Let $a, c>0, b \geq 0, d \leq 0$. The transformation with

$$
\left(\begin{array}{ll}
a & b \\
c & d
\end{array}\right)
$$


transforms the FBSDE (1.1) into an FBSDE satisfying SLC if and only if the transformation with

$$
\left(\begin{array}{ll}
1 & \frac{b}{a} \\
1 & \frac{d}{c}
\end{array}\right)
$$

does so.

Proof. Let $\hat{\sigma}, \hat{\xi}, \Phi$ be defined as above, and $\tilde{\sigma}$ and $\tilde{\xi}$ be the diffusion coefficient and terminal condition of the transformed FBDSE with $\tilde{a}:=1, \tilde{b}:=\frac{b}{a}, \tilde{c}:=1, \tilde{d}:=\frac{d}{c}$. Assume that $L_{\tilde{\xi}, x}<L_{\tilde{\sigma}, z}^{-1}$. Then we obtain that

$$
\begin{aligned}
\left|\frac{\partial \hat{\sigma}}{\partial z}(s, x, y, z)\right| & =\left|\frac{a \frac{\partial \sigma}{\partial z}(s, \Phi(s, x, y, z))+b}{c \frac{\partial \sigma}{\partial z}(s, \Phi(s, x, y, z))+d}\right|=\frac{a}{c}\left|\frac{\partial \tilde{\sigma}}{\partial z}\left(s, \frac{x}{a}, \frac{y}{c}, z\right)\right| \leq \frac{a}{c} L_{\tilde{\sigma}, z}, \\
\left|\hat{\xi}^{\prime}(x)\right| & =\left|\frac{c+d \xi^{\prime}\left((a \operatorname{Id}+b \xi)^{-1}(x)\right)}{a+b \xi^{\prime}\left((a \operatorname{Id}+b \xi)^{-1}(x)\right)}\right|=\frac{c}{a}\left|\tilde{\xi}^{\prime}\left(\left(\operatorname{Id}+\frac{b}{a} \xi\right)(x)\right)\right| \leq \frac{c}{a} L_{\tilde{\xi}, x} .
\end{aligned}
$$

Consequently, $L_{\hat{\xi}, x} \leq \frac{c}{a} L_{\tilde{\xi}, x}<\frac{c}{a} L_{\tilde{\sigma}, z}^{-1} \leq L_{\hat{\sigma}, z}^{-1}$. The converse implication follows analogously.

For the remainder of this section let $a, c=1$. To gain standard Lipschitz conditions for the transformed FBSDE (2.2) we have to choose $b$ and $d$ depending on $\xi$ and $\sigma$. We derive for which choice the derivatives of $\hat{\xi}$ and $\hat{\sigma}$ are bounded such that $L_{\hat{\xi}, x}<L_{\hat{\sigma}, z}^{-1}$. Note that one can rewrite $\hat{\sigma}$ as

$$
\hat{\sigma}(s, x, y, z)=z+(b-d) \hat{\varphi}(s, x, y, z) \text {. }
$$

We observe that

$$
\frac{\partial \hat{\sigma}}{\partial z}(s, x, y, z)=1+\frac{b-d}{\frac{\partial \sigma}{\partial z}(s, \hat{\varphi}(s, x, y, z))+d}=1-\frac{b-d}{-\frac{\partial \sigma}{\partial z}(s, \hat{\varphi}(s, x, y, z))-d}
$$

and

$$
\hat{\xi}^{\prime}(x)=\frac{1+d \xi^{\prime}\left((\mathrm{Id}+b \xi)^{-1}(x)\right)}{1+b \xi^{\prime}\left((\operatorname{Id}+b \xi)^{-1}(x)\right)}
$$

for all $x, y, z \in \mathbb{R}, s \in[0, T]$. Note that the denominator $-\frac{\partial \sigma}{\partial z}(s, x, y, z)-d$ in $(2.3)$ is always not equal to zero because of (ii), and we obtain the desired estimate if we choose $d \leq 0$ close enough to zero and the constant $b$ as a positive real number. To this end, let $d \in\left[-L_{\xi, x}^{-1}, 0\right]$ such that $-d+K_{\sigma, z}>0$, and $b \in\left[0,-d \vee K_{\sigma, z}\right)$. With this choice of $b$ and $d$ we have for all $x, y, z \in \mathbb{R}, s \in[0, T]$ either

$$
\left|\frac{\partial \hat{\sigma}}{\partial z}(s, x, y, z)\right|=1-\frac{b-d}{-\frac{\partial \sigma}{\partial z}\left(s, \frac{d x-b y}{d-b}, \frac{-x+y}{d-b}, z\right)-d} \leq 1-\frac{b-d}{L_{\sigma, z}-d}<1,
$$

or

$$
\begin{aligned}
\left|\frac{\partial \hat{\sigma}}{\partial z}(s, x, y, z)\right| & =\frac{b-d}{-\frac{\partial \sigma}{\partial z}(s, x, y, z)-d}-1 \leq \frac{b+\left(-d \vee K_{\sigma, z}\right)}{-d \vee K_{\sigma, z}}-1 \\
& \leq \frac{b}{-d \vee K_{\sigma, z}}<1,
\end{aligned}
$$

and consequently $L_{\hat{\sigma}, z}<1$. Similarly, we deduce that for all $x \in \mathbb{R}$

$$
\left|\hat{\xi}^{\prime}(x)\right|=\frac{1+d \xi^{\prime}\left((\mathrm{Id}+b \xi)^{-1}(x)\right)}{1+b \xi^{\prime}\left((\operatorname{Id}+b \xi)^{-1}(x)\right)} \leq 1
$$

and thus $L_{\hat{\xi}, x} \leq 1$. 


\section{Decoupling fields}

A so-called decoupling field to an FBSDE comes with a richer structure than just a classical solution $(X, Y, Z)$ to $(1.1)$.

Definition 3.1. Let $t \in[0, T]$. A function $u: \Omega \times[t, T] \times \mathbb{R} \rightarrow \mathbb{R}$ with $u(T, \cdot)=\xi$ a.e. is called decoupling field for $(\xi, \mu, \sigma, f)$ on $[t, T]$ if for all $t_{1}, t_{2} \in[t, T]$ with $t_{1} \leq t_{2}$ and any $\mathcal{F}_{t_{1}}$-measurable $X_{t_{1}}: \Omega \rightarrow \mathbb{R}$ there exist progressively measurable processes $(X, Y, Z)$ on $\left[t_{1}, t_{2}\right]$ such that

$$
\begin{aligned}
& X_{s}=X_{t_{1}}+\int_{t_{1}}^{s} \mu\left(r, X_{r}, Y_{r}, Z_{r}\right) d r+\int_{t_{1}}^{s} \sigma\left(r, X_{r}, Y_{r}, Z_{r}\right) d W_{r}, \\
& Y_{s}=Y_{t_{2}}-\int_{s}^{t_{2}} f\left(r, X_{r}, Y_{r}, Z_{r}\right) d r-\int_{s}^{t_{2}} Z_{r} d W_{r}, \\
& Y_{s}=u\left(s, X_{s}\right), \quad \text { (decoupling condition) }
\end{aligned}
$$

a.s. for all $s \in\left[t_{1}, t_{2}\right]$. In particular, we want all integrals to be well-defined.

For the following we need to introduce further notation: Let $I \subseteq[0, T]$ be an interval and $u: \Omega \times I \times \mathbb{R} \rightarrow \mathbb{R}$ be a map such that $u(s, \cdot)$ is measurable for every $s \in I$. We define

$$
\begin{aligned}
& L_{u, x}:=\sup _{s \in I} \inf \{L \geq 0 \mid \text { for a.a. } \omega \in \Omega: \\
& \left.\qquad\left|u(\omega, s, x)-u\left(\omega, s, x^{\prime}\right)\right| \leq L\left|x-x^{\prime}\right| \text { for all } x, x^{\prime} \in \mathbb{R}\right\},
\end{aligned}
$$

where $\inf \emptyset:=\infty$. We also set $L_{u, x}:=\infty$ if $u(s, \cdot)$ is not measurable for every $s \in I$. One can show that $L_{u, x}<\infty$ is equivalent to $u$ having a modification which is truly Lipschitz continuous in $x \in \mathbb{R}$. Indeed, it is enough to redefine $u(s, \omega, \cdot)=0$ whenever Lipschitz continuity is not satisfied (see Lemma 2.1.3. in [7]).

Definition 3.2. Let $u: \Omega \times[t, T] \times \mathbb{R} \rightarrow \mathbb{R}$ be a decoupling field for $(\xi, \mu, \sigma, f)$. We say $u$ to be weakly regular if $L_{u, x}<L_{\sigma, z}^{-1}$ and $\sup _{s \in[t, T]}\|u(\cdot, s, 0)\|_{\infty}<\infty$.

Definition 3.3. We define the maximal interval $I_{\max } \subseteq[0, T]$ of the problem given by $(\xi, \mu, \sigma, f)$ as the union of all intervals $[t, T] \subseteq[0, T]$, such that there exists a weakly regular decoupling field $u$ on $[t, T]$.

Note that the maximal interval might be open to the left. Also, let us remark that we define a decoupling field on such an interval as a mapping which is a decoupling field on every compact subinterval containing $T$. Similarly we can define weakly regular decoupling fields as mappings which restricted to an arbitrary compact subinterval containing $T$ are weakly regular decoupling fields in the sense of the definition given above.

Finally, we have global existence and uniqueness on the maximal interval:

Theorem 3.4 ([7], Theorem 5.1.11, Lemma 5.1.12 and Corollary 2.5.5). Assume that $(\xi, \mu, \sigma, f)$ satisfy $S L C$. Then there exists a unique weakly regular decoupling field 
$u$ on $I_{\max }$. Furthermore, either $I_{\max }=[0, T]$ or $I_{\max }=\left(t_{\min }, T\right]$, where $0 \leq t_{\min }<T$. In the latter case we have

$$
\lim _{t \downarrow t_{\min }} L_{u(t, \cdot), x}=L_{\sigma, z}^{-1}
$$

Moreover, for any $t \in I_{\max }$ and any initial condition $X_{t}=x \in \mathbb{R}$ there is a unique solution $(X, Y, Z)$ of the FBSDE (1.1) on $[t, T]$ satisfying

$$
\sup _{s \in[t, T]} \mathbb{E}\left[\left|X_{s}\right|^{2}\right]+\sup _{s \in[t, T]} \mathbb{E}\left[\left|Y_{s}\right|^{2}\right]+\mathbb{E}\left[\int_{t}^{T}\left|Z_{s}\right|^{2} d s\right]<\infty .
$$

This $(X, Y, Z)$ satisfies the decoupling condition $Y_{s}=u\left(s, X_{s}\right), s \in[t, T]$.

\section{Main results}

In this section we prove that the transformation derived in section 2 turns FBSDE (1.1) into an FBSDE satisfying SLC. Recall the definition of the constants $K_{\sigma, z}, K_{\xi, x}$ in $(2.1)$ and let $\kappa \in\left[0, L_{\xi, x}^{-1}\right] \cap \mathbb{R}$ and $\gamma \in\left[0, \kappa \vee K_{\sigma, z}\right]$, such that

- $\kappa+\gamma>0$

- $\kappa+K_{\sigma, z}>0$, and

- $\gamma<\kappa \vee K_{\sigma, z}$ if $K_{\xi, x}=0$.

We consider the transformation with $c:=\gamma$ and $d:=-\kappa$, that means

$$
\left(\begin{array}{c}
\hat{X}_{t} \\
\hat{Y}_{t}
\end{array}\right):=\left(\begin{array}{cc}
1 & \gamma \\
1 & -\kappa
\end{array}\right)\left(\begin{array}{c}
X_{t} \\
Y_{t}
\end{array}\right)
$$

and $\hat{Z}_{t}:=\sigma\left(t, X_{t}, Y_{t}, Z_{t}\right)-\kappa Z_{t}$ for $t \in[0, T]$.

Remark 4.1. In the case $L_{\xi, x}>0$ all the reasoning in this section is also true if we choose $\kappa:=L_{\xi, x}^{-1}$ and $\gamma:=\frac{\kappa}{2}$. However, in this section we prove the results in a more general setting and leave some freedom in the choice of $\kappa$ and $\gamma$ by just specifying an interval to which the constants should belong. This enables us to choose $\kappa=0$ in the case where $\sigma$ is strictly decreasing in $z$ with a slope of at least $K_{\sigma, z}>0$, which simplifies the transformation. Note that if $K_{\sigma, z}=0$ we have to choose a positive $\kappa$ in order to obtain a Lipschitz continuous diffusion coefficient $\hat{\sigma}$.

The auxiliary FBSDE takes the form

$$
\begin{aligned}
& \hat{X}_{t}=\hat{x}+\int_{0}^{t} \hat{\mu}\left(s, \hat{X}_{s}, \hat{Y}_{s}, \hat{Z}_{s}\right) d s+\int_{0}^{t} \hat{\sigma}\left(s, \hat{X}_{s}, \hat{Y}_{s}, \hat{Z}_{s}\right) d W_{s}, \\
& \hat{Y}_{t}=\hat{\xi}\left(\hat{X}_{T}\right)-\int_{t}^{T} \hat{f}\left(s, \hat{X}_{t}, \hat{Y}_{s}, \hat{Z}_{s}\right) d s-\int_{t}^{T} \hat{Z}_{s} d W_{s}, t \in[0, T],
\end{aligned}
$$


where the parameter functions are given by

$$
\begin{aligned}
\hat{\mu}(s, x, y, z) & :=\mu(s, \Phi(s, x, y, z))+\gamma f(s, \Phi(s, x, y, z)), \\
\hat{\sigma}(s, x, y, z) & :=z+(\kappa+\gamma) \hat{\varphi}(s, x, y, z), \\
\hat{f}(s, x, y, z) & :=\mu(s, \Phi(s, x, y, z))-\kappa f(s, \Phi(s, x, y, z)), \\
\hat{\xi}(x) & :=\left((\operatorname{Id}-\kappa \xi) \circ(\operatorname{Id}+\gamma \xi)^{-1}\right)(x),
\end{aligned}
$$

with

$$
\begin{aligned}
\varphi(s, x, y, z) & :=(\sigma(s, x, y, \cdot)-\kappa \mathrm{Id})^{-1}(z), \\
\hat{\varphi}(s, x, y, z) & :=\varphi\left(s, \frac{\kappa x+\gamma y}{\kappa+\gamma}, \frac{x-y}{\kappa+\gamma}, z\right), \\
\Phi(s, x, y, z) & :=\left(\frac{\kappa x+\gamma y}{\kappa+\gamma}, \frac{x-y}{\kappa+\gamma}, \hat{\varphi}(s, x, y, z)\right),
\end{aligned}
$$

for all $s \in[0, T], x, y, z \in \mathbb{R}$.

We now state the two main results of this paper.

Proposition 4.2. The parameters $(\hat{\xi}, \hat{\mu}, \hat{\sigma}, \hat{f})$ satisfy $S L C$. Moreover, for the parameters $(\hat{\xi}, \hat{\mu}, \hat{\sigma}, \hat{f})$ there exists a maximal interval $I_{\max } \subseteq[0, T]$ of the form $[0, T]$ or $\left(t_{\min }, T\right]$ and a decoupling field $\hat{u}: \Omega \times I_{\max } \times \mathbb{R} \rightarrow \mathbb{R}$ that is weakly regular.

Theorem 4.3. Let $I_{\max }$ be the maximal interval and $\hat{u}$ be a weakly regular decoupling field for the parameters $(\hat{\xi}, \hat{\mu}, \hat{\sigma}, \hat{f})$. Fix $t \in I_{\max }$ and assume that the mapping $\mathbb{R} \rightarrow \mathbb{R}, x \mapsto(\kappa I d+\gamma \hat{u}(t, \cdot))(x)$ is bijective. Then for any initial value $x \in \mathbb{R}$ there exists a solution $(X, Y, Z)$ to FBSDE (1.1) on the interval $[t, T]$.

Moreover, if $\mathbb{R} \rightarrow \mathbb{R}, x \mapsto(\kappa I d+\gamma \hat{u}(s, \cdot))(x)$ is bijective for all $s \in I_{\max }$ the function $u: \Omega \times I_{\max } \times \mathbb{R} \rightarrow \mathbb{R}$, defined by

$$
u(s, x):=\frac{1}{\kappa+\gamma}\left((I d-\hat{u}(s, \cdot)) \circ(\kappa I d+\gamma \hat{u}(s, \cdot))^{-1}\right)((\kappa+\gamma) x),
$$

is a decoupling field for $(\xi, \mu, \sigma, f)$.

Corollary 4.4. If $I_{\max }=[0, T]$ and the mapping $\mathbb{R} \rightarrow \mathbb{R}, x \mapsto(\kappa I d+\gamma \hat{u}(0, \cdot))(x)$ is bijective, then for any initial value $x \in \mathbb{R}$ there exists a unique solution $(X, Y, Z)$ to FBSDE (1.1) on the interval $[0, T]$ such that (3.3) is satisfied for $t=0$.

Remark 4.5. Concerning Theorem 4.3 we mention the following:

(a) To prove that $I_{\max }=[0, T]$ one can apply the method of decoupling fields to the auxiliary FBSDE (4.1). To this end, one studies the dynamics of the gradient process $V_{t}=\partial_{x} \hat{u}\left(t, \hat{X}_{t}\right)$ and shows that $V_{t}$ can be bounded away from $L_{\sigma, z}^{-1}$ uniformly on $I_{\max }$. Then Theorem 3.4 implies that only $I_{\max }=[0, T]$ is possible. Finally, Corollary 4.4 implies that also the original FBSDE (1.1) has a global solution if the mapping $\mathbb{R} \rightarrow \mathbb{R}, x \mapsto(\kappa \mathrm{Id}+\gamma \hat{u}(0, \cdot))(x)$ is bijective. 
(b) The bijectivity of the mapping $\mathbb{R} \rightarrow \mathbb{R}, x \mapsto(\kappa \operatorname{Id}+\gamma \hat{u}(t, \cdot))(x)$ for some fixed $t \in I_{\max }$ is necessary to establish for an arbitrary $x \in \mathbb{R}$ the existence of an appropriate initial value $(\kappa \mathrm{Id}+\gamma \hat{u}(t, \cdot))^{-1}((\kappa+\gamma) x)$ for the auxiliary FBSDE (4.1). A solution of the auxiliary FBSDE with this initial value provides a solution to the original FBSDE (1.1) with initial value $x$ after the backward transformation (see Lemma 4.6 below).

(c) To prove that the mapping $\mathbb{R} \rightarrow \mathbb{R}, x \mapsto(\kappa \operatorname{Id}+\gamma \hat{u}(t, \cdot))(x)$ is bijective for some fixed $t \in I_{\max }$ can be an involving task. Indeed, there are cases where we have global solvability of the transformed FBSDE, but the bijectivity condition fails and hence we cannot conclude global solvability of our original FBSDE. This problem is presented in section 5.2, where one can even derive an explicit formula for the decoupling field $\hat{u}$. Nevertheless, one does not always need an explicit representation of $\hat{u}$ at the initial time. It can be enough to bound the Lipschitz constant of $\hat{u}$ in an appropriate way, e.g. with the method of decoupling fields. With an adequate bound the bijectivity can follow right away. Since the method of decoupling fields is primarily used to show global solvability (see (a)) the bijectivity can follow as a byproduct (see section 5.3).

In order to prove Proposition 4.2, Theorem 4.3 and Corollary 4.4, we show at first that a solution to our original FBSDE (1.1) can be recovered from the transformed FBSDE (4.1). The proofs can be found at pages $14-17$.

Lemma 4.6. If there exists a solution $(\hat{X}, \hat{Y}, \hat{Z})$ to the FBSDE (4.1) on a time interval $[t, T]$ for some $t \in[0, T)$ with an $\mathcal{F}_{t}$-measurable initial condition $\hat{x}$, then $(X, Y, Z)$, defined as

$$
\begin{aligned}
\left(\begin{array}{c}
X_{s} \\
Y_{s}
\end{array}\right) & :=\frac{1}{\kappa+\gamma}\left(\begin{array}{cc}
\kappa & \gamma \\
1 & -1
\end{array}\right)\left(\begin{array}{c}
\hat{X}_{s} \\
\hat{Y}_{s}
\end{array}\right) \\
Z_{s} & :=\hat{\varphi}\left(s, \hat{X}_{s}, \hat{Y}_{s}, \hat{Z}_{s}\right)=\left(\sigma\left(s, X_{s}, Y_{s}, \cdot\right)-\kappa I d\right)^{-1}\left(\hat{Z}_{s}\right), s \in[t, T],
\end{aligned}
$$

solves the FBSDE (1.1) on $[t, T]$ with the $\mathcal{F}_{t}$-measurable initial condition $\frac{\kappa \hat{x}+\gamma \hat{Y}_{t}}{\kappa+\gamma}$.

Proof. We verify that $(X, Y, Z)$, defined as above, fulfills the FBSDE (1.1). To this end, note that $\hat{\varphi}\left(s, \hat{X}_{s}, \hat{Y}_{s}, \hat{Z}_{s}\right)=Z_{s}$ and $\Phi\left(s, \hat{X}_{s}, \hat{Y}_{s}, \hat{Z}_{s}\right)=\left(X_{s}, Y_{s}, Z_{s}\right)$. Consequently,

$$
\begin{aligned}
d X_{s}= & \frac{1}{\kappa+\gamma}\left(\kappa d \hat{X}_{s}+\gamma d \hat{Y}_{s}\right) \\
= & \frac{\kappa}{\kappa+\gamma}\left(\hat{\mu}\left(s, \hat{X}_{s}, \hat{Y}_{s}, \hat{Z}_{s}\right) d s+\hat{\sigma}\left(s, \hat{X}_{s}, \hat{Y}_{s}, \hat{Z}_{s}\right) d W_{s}\right) \\
& \quad+\frac{\gamma}{\kappa+\gamma}\left(\hat{f}\left(s, \hat{X}_{s}, \hat{Y}_{s}, \hat{Z}_{s}\right) d s+\hat{Z}_{s} d W_{s}\right) \\
= & \mu\left(s, X_{s}, Y_{s}, Z_{s}\right) d s+\sigma\left(s, X_{s}, Y_{s}, Z_{s}\right) d W_{s},
\end{aligned}
$$


and

$$
\begin{aligned}
d Y_{s}= & \frac{1}{\kappa+\gamma}\left(d \hat{X}_{s}-d \hat{Y}_{s}\right) \\
= & \frac{1}{\kappa+\gamma}\left[\left(\hat{\mu}\left(s, \hat{X}_{s}, \hat{Y}_{s}, \hat{Z}_{s}\right)-\hat{f}\left(s, \hat{X}_{s}, \hat{Y}_{s}, \hat{Z}_{s}\right)\right) d s\right. \\
& \left.\quad+\left(\hat{\sigma}\left(s, \hat{X}_{s}, \hat{Y}_{s}, \hat{Z}_{s}\right)-\hat{Z}_{s}\right) d W_{s}\right] \\
& =f\left(s, X_{s}, Y_{s}, Z_{s}\right) d s+Z_{s} d W_{s}
\end{aligned}
$$

We observe that $X_{t}=\frac{\kappa \hat{x}+\gamma \hat{Y}_{t}}{\kappa+\gamma}$ by the same calculations as above. If $\gamma=0$ we have $X=\hat{X}, \hat{\xi}(x)=x-\kappa \xi(x)$ and therefore

$$
Y_{T}=\frac{1}{\kappa}\left(\hat{X}_{T}-\hat{Y}_{T}\right)=\frac{1}{\kappa}\left(\hat{X}_{T}-\hat{\xi}\left(\hat{X}_{T}\right)\right)=\xi\left(X_{T}\right), \text { a.s. }
$$

If $\gamma>0$ we can rewrite $\hat{\xi}$ in a different form, because

$$
\begin{aligned}
\hat{\xi}(x) & =\left((\operatorname{Id}-\kappa \xi) \circ(\operatorname{Id}+\gamma \xi)^{-1}\right)(x) \\
& =(\operatorname{Id}+\gamma \xi)^{-1}(x)-\kappa \xi\left((\operatorname{Id}+\gamma \xi)^{-1}(x)\right) \\
& =(\operatorname{Id}+\gamma \xi)^{-1}(x)-\frac{\kappa}{\gamma}(\operatorname{Id}+\gamma \xi)\left((\operatorname{Id}+\gamma \xi)^{-1}(x)\right)+\frac{\kappa}{\gamma}(\operatorname{Id}+\gamma \xi)^{-1}(x) \\
& =\frac{\kappa+\gamma}{\gamma}(\operatorname{Id}+\gamma \xi)^{-1}(x)-\frac{\kappa}{\gamma} x, x \in \mathbb{R} .
\end{aligned}
$$

We obtain

$$
\begin{aligned}
Y_{T} & =\frac{1}{\kappa+\gamma}\left(\hat{X}_{T}-\hat{Y}_{T}\right)=\frac{1}{\kappa+\gamma}\left(\hat{X}_{T}-\hat{\xi}\left(\hat{X}_{T}\right)\right) \\
& =\frac{1}{\kappa+\gamma}\left(\hat{X}_{T}-\frac{\kappa+\gamma}{\gamma}(\operatorname{Id}+\gamma \xi)^{-1}\left(\hat{X}_{T}\right)+\frac{\kappa}{\gamma} \hat{X}_{T}\right) \\
& =\frac{1}{\gamma}\left(\hat{X}_{T}-(\operatorname{Id}+\gamma \xi)^{-1}\left(\hat{X}_{T}\right)\right) \\
& =\frac{1}{\gamma}\left((\operatorname{Id}+\gamma \xi)\left((\operatorname{Id}+\gamma \xi)^{-1}\left(\hat{X}_{T}\right)\right)-(\operatorname{Id}+\gamma \xi)^{-1}\left(\hat{X}_{T}\right)\right) \\
& =\xi\left((\operatorname{Id}+\gamma \xi)^{-1}\left(\hat{X}_{T}\right)\right)=\xi\left(X_{T}\right), \text { a.s., }
\end{aligned}
$$

by an alternative representation of $\hat{\xi}$ and because

$$
\begin{aligned}
(\operatorname{Id}+\gamma \xi)^{-1}\left(\hat{X}_{T}\right) & =\frac{1}{\kappa+\gamma}\left[\kappa \hat{X}_{T}+(\kappa+\gamma)(\operatorname{Id}+\gamma \xi)^{-1}\left(\hat{X}_{T}\right)-\kappa \hat{X}_{T}\right] \\
& =\frac{1}{\kappa+\gamma}\left[\kappa \hat{X}_{T}+\gamma \hat{\xi}\left(\hat{X}_{T}\right)\right]=\frac{1}{\kappa+\gamma}\left[\kappa \hat{X}_{T}+\gamma \hat{Y}_{T}\right]=X_{T} \text {, a.s. }
\end{aligned}
$$

This concludes the proof.

Now we show that the function $\varphi$ is Lipschitz continuous with the help of an inverse function theorem for Lipschitz functions. Later on we use this result for proving that the parameters $(\hat{\xi}, \hat{\mu}, \hat{\sigma}, \hat{f})$ fulfill SLC. 
Lemma 4.7. The function $\varphi$ is Lipschitz continuous in $(x, y, z)$ and we have for the Lipschitz constant of $\varphi$ in $z$ that $L_{\varphi, z}=\frac{1}{\kappa+K_{\sigma, z}}$. Moreover, we have

$$
\left|\varphi\left(t, x, y, z_{1}\right)-\varphi\left(t, x, y, z_{2}\right)\right| \geq \frac{1}{L_{\sigma, z}+\kappa}\left|z_{1}-z_{2}\right|
$$

for all $x, y, z_{1}, z_{2} \in \mathbb{R}$ and $t \in[0, T]$. In particular, $\hat{\varphi}$ is Lipschitz continuous in $(x, y, z)$ and $(4.4)$ holds also true for $\hat{\varphi}$.

Proof. Let $(\omega, t) \in \Omega \times[0, T]$. At first, we prove the Lipschitz continuity of $\varphi$ in $z$ and that (4.4) holds true. To this end, let $x, y \in \mathbb{R}$ be fixed for the moment. Note that $\sigma(t, x, y, \cdot)-\kappa \mathrm{Id}$ is strictly decreasing and satisfies

$\left(\kappa+K_{\sigma, z}\right)\left|z_{1}-z_{2}\right| \leq\left|\sigma\left(t, x, y, z_{1}\right)-\kappa z_{1}-\left(\sigma\left(t, x, y, z_{2}\right)-\kappa z_{2}\right)\right| \leq\left(\kappa+L_{\sigma, z}\right)\left|z_{1}-z_{2}\right|$

for all $z_{1}, z_{2} \in \mathbb{R}$. Hence, $\sigma(t, x, y, \cdot)-\kappa$ Id is a bijection from $\mathbb{R}$ onto $\mathbb{R}$ and the inverse $\varphi(t, x, y, \cdot)$ is also Lipschitz continuous, because it satisfies

$$
\frac{1}{\kappa+L_{\sigma, z}}\left|z_{1}-z_{2}\right| \leq\left|\varphi\left(t, x, y, z_{1}\right)-\varphi\left(t, x, y, z_{2}\right)\right| \leq \frac{1}{\kappa+K_{\sigma, z}}\left|z_{1}-z_{2}\right|
$$

for all $z_{1}, z_{2} \in \mathbb{R}$. That means (4.4) holds true and $L_{\varphi, z}=\frac{1}{\kappa+K_{\sigma, z}}$ due to the definition of $K_{\sigma, z}$.

It remains to show the Lipschitz continuity of $\varphi(t, \cdot, \cdot, z)$. The map $(x, y, z) \mapsto$ $\sigma(t, x, y, z)-\kappa z$ is Lipschitz continuous and therefore differentiable almost everywhere. In particular, for all $x, y \in \mathbb{R}$ we have

$$
\frac{\partial \sigma}{\partial z}(t, x, y, z)-\kappa \leq-K_{\sigma, z}-\kappa<0
$$

for a.e. $z \in \mathbb{R}$, since $\sigma$ is monotonically decreasing due to (A5). Our goal is to apply Theorem 1 in [4] to show that $\varphi$ is Lipschitz continuous. To this end, we define the mapping

$$
F: \mathbb{R}^{3} \rightarrow \mathbb{R}^{3},(x, y, z) \mapsto(x, y, \sigma(t, x, y, z)-\kappa z),
$$

which has the inverse $F^{-1}(x, y, z)=(x, y, \varphi(t, x, y, z))$. Let $\left(x_{0}, y_{0}, z_{0}\right) \in \mathbb{R}^{3}$ and $a_{0}:=\varphi\left(t, x_{0}, y_{0}, z_{0}\right)$. We prove that there exists a neighbourhood of $\left(x_{0}, y_{0}, z_{0}\right)$ on which $\varphi$ is Lipschitz continuous and that the derivatives are bounded almost everywhere.

Note that for Clarke's generalized Jacobian $\partial F\left(x_{0}, y_{0}, a_{0}\right)$ of $F$ in $\left(x_{0}, y_{0}, a_{0}\right)$ we have

$$
\partial F\left(x_{0}, y_{0}, a_{0}\right) \subseteq\left\{\left(\begin{array}{ccc}
1 & 0 & 0 \\
0 & 1 & 0 \\
\lambda_{1} & \lambda_{2} & \lambda_{3}
\end{array}\right):\left|\lambda_{1}\right|,\left|\lambda_{2}\right| \leq L, \lambda_{3} \leq-K_{\sigma, z}-\kappa<0\right\}
$$

see Definition 1 in [4] for details. Hence, every element of $\partial F\left(x_{0}, y_{0}, a_{0}\right)$ has full rank and the requirements of Theorem 1 in [4] are met. Thus, there exist neighbourhoods $U$ and $V$ of $\left(x_{0}, y_{0}, a_{0}\right)$ and $F\left(x_{0}, y_{0}, a_{0}\right)=\left(x_{0}, y_{0}, z_{0}\right)$, respectively, and a Lipschitz 
continuous function $G: V \rightarrow U$ such that $F \circ G=\operatorname{Id}_{V}$ and $G \circ\left(\left.F\right|_{U}\right)=\operatorname{Id}_{U}$. We observe that

$$
G(x, y, z)=F^{-1}(x, y, z)=(x, y, \varphi(t, x, y, z))
$$

for all $(x, y, z) \in V$, because we have already identified the inverse of $F$ above. The Lipschitz continuity of $F$ and $G$ implies that:

- $\varphi$ is Lipschitz continuous in $(x, y, z)$ on $V$, and therefore, there exists a null set $N_{1} \subseteq V$ such that $\varphi$ is differentiable on $V \backslash N_{1}$,

- there exists a null set $N_{2} \subseteq U$ such that $F$ is differentiable on $U \backslash N_{2}$,

- $F$ and $G$ satisfy Lusin's property (N), i.e. they map null sets onto null sets, which implies that $F\left(N_{2}\right) \subseteq V$ and $G\left(N_{1}\right)=F^{-1}\left(N_{1}\right) \subseteq U$ are null sets (see e.g. [3, p. 194] for a definition).

These properties entail that $\varphi \circ F$ is also differentiable on $U \backslash\left(N_{2} \cup F^{-1}\left(N_{1}\right)\right)$ according to the chain rule, that means $\varphi \circ F$ is differentiable almost everywhere. To show that the partial derivatives of $\varphi$ are essentially bounded, let $(x, y, z) \in$ $V \backslash\left(N_{1} \cup F\left(N_{2}\right)\right)$. The chain rule applied to the identity $\varphi \circ F(x, y, a)=a$ with $a:=\varphi(x, y, z)$ yields that

$$
\begin{aligned}
& \frac{\partial \varphi}{\partial x}(t, x, y, z)=-\frac{\frac{\partial \sigma}{\partial x}(t, x, y, a)}{\frac{\partial \sigma}{\partial z}(t, x, y, a)-\kappa}, \\
& \frac{\partial \varphi}{\partial y}(t, x, y, z)=-\frac{\frac{\partial \sigma}{\partial y}(t, x, y, a)}{\frac{\partial \sigma}{\partial z}(t, x, y, a)-\kappa}, \\
& \frac{\partial \varphi}{\partial z}(t, x, y, z)=\frac{1}{\frac{\partial \sigma}{\partial z}(t, x, y, a)-\kappa},
\end{aligned}
$$

because $(x, y, a) \in U \backslash\left(N_{2} \cup F^{-1}\left(N_{1}\right)\right)$. The essential boundedness of the derivatives follows since $\left|\frac{\partial \sigma}{\partial x}(t, x, y, a)\right|,\left|\frac{\partial \sigma}{\partial y}(t, x, y, a)\right| \leq L$ by $(\mathrm{A} 2),\left|\frac{\partial \sigma}{\partial z}(t, x, y, a)-\kappa\right| \geq K_{\sigma, z}+\kappa$ by (4.5), and because the set $V \backslash\left(N_{1} \cup F\left(N_{2}\right)\right)$ has full measure. Consequently, the function $\varphi(t, \cdot, \cdot, \cdot)$ is Lipschitz continuous on $V$ with a Lipschitz constant $M$ smaller than or equal to $\frac{\sqrt{3} L}{K_{\sigma, z}+\kappa}$. Since in the beginning the choice of $\left(x_{0}, y_{0}, z_{0}\right)$ was arbitrary, the mapping $\varphi(t, \cdot, \cdot, \cdot): \mathbb{R}^{3} \rightarrow \mathbb{R}$ is locally Lipschitz continuous with Lipschitz constant $M$. Finally, we obtain the global Lipschitz continuity of $\varphi$ in $(x, y, z)$ with Lipschitz constant $M$.

With the help of the two lemmas above we are able to prove Proposition 4.2, Theorem 4.3 and Corollary 4.4.

Proof of Proposition 4.2. The Lipschitz continuity of $\hat{\mu}$ and $\hat{f}$ is obvious because those functions are compositions of Lipschitz continuous functions (note in particular Lemma 4.7).

Now we examine the terminal condition $\hat{\xi}$ and the diffusion coefficient $\hat{\sigma}$. Let $(\omega, t) \in \Omega \times[0, T]$. Then 
1. Lipschitz continuity of $\hat{\xi}$ : $(\mathrm{Id}+\gamma \xi)$ is strictly increasing such that

$$
\left(1+\gamma K_{\xi, x}\right)\left|x_{1}-x_{2}\right| \leq\left|(\operatorname{Id}+\gamma \xi)\left(x_{1}\right)-(\operatorname{Id}+\gamma \xi)\left(x_{2}\right)\right|
$$

for all $x_{1}, x_{2} \in \mathbb{R}$. Therefore, $(\mathrm{Id}+\gamma \xi)^{-1}$ is also Lipschitz continuous with $L_{(\operatorname{Id}+\gamma \xi)^{-1}, x} \leq \frac{1}{1+\gamma K_{\xi, x}}$. The function (Id $\left.-\kappa \xi\right)$ is strictly increasing due to the definition of $\kappa$, because for all $x_{1}, x_{2} \in \mathbb{R}, x_{1} \leq x_{2}$,

$$
(\operatorname{Id}-\kappa \xi)\left(x_{2}\right)-(\operatorname{Id}-\kappa \xi)\left(x_{1}\right) \geq\left(1-\kappa L_{\xi, x}\right)\left(x_{2}-x_{1}\right) \geq 0 .
$$

Moreover, it is Lipschitz continuous with $L_{(\mathrm{Id}-\kappa \xi), x} \leq 1$. We conclude that $\hat{\xi}$ is Lipschitz continuous with $L_{\hat{\xi}, x} \leq \frac{1}{1+\gamma K_{\xi, x}}$. In more detail, we have $L_{\hat{\xi}, x} \leq 1$ if $K_{\xi, x}=0$, and $L_{\hat{\xi}, x}<1$ if $K_{\xi, x}>0$.

2. Lipschitz continuity of $\hat{\sigma}$ : The Lipschitz continuity of $\hat{\sigma}$ in $(x, y)$ is clear since $\hat{\varphi}$ is Lipschitz continuous according to Lemma 4.7. Let $x, y \in \mathbb{R}$ and $z_{1}, z_{2} \in \mathbb{R}$, $z_{1}<z_{2}$. Then either

$$
\begin{aligned}
\left|\hat{\sigma}\left(t, x, y, z_{1}\right)-\hat{\sigma}\left(t, x, y, z_{2}\right)\right| & =\hat{\sigma}\left(t, x, y, z_{1}\right)-\hat{\sigma}\left(t, x, y, z_{2}\right) \\
& =z_{1}-z_{2}+(\gamma+\kappa)\left(\hat{\varphi}\left(t, x, y, z_{1}\right)-\hat{\varphi}\left(t, x, y, z_{2}\right)\right) \\
& \leq\left(\frac{\gamma+\kappa}{\kappa+K_{\sigma, z}}-1\right)\left(z_{2}-z_{1}\right)=\frac{\gamma-K_{\sigma, z}}{\kappa+K_{\sigma, z}}\left|z_{1}-z_{2}\right|
\end{aligned}
$$

or

$$
\begin{aligned}
\left|\hat{\sigma}\left(t, x, y, z_{1}\right)-\hat{\sigma}\left(t, x, y, z_{2}\right)\right| & =\hat{\sigma}\left(t, x, y, z_{2}\right)-\hat{\sigma}\left(t, x, y, z_{1}\right) \\
& =z_{2}-z_{1}-(\gamma+\kappa)\left(\hat{\varphi}\left(t, x, y, z_{1}\right)-\hat{\varphi}\left(t, x, y, z_{2}\right)\right) \\
& \leq\left(1-\frac{\gamma+\kappa}{L_{\sigma, z}+\kappa}\right)\left(z_{2}-z_{1}\right)
\end{aligned}
$$

holds true. We obtain that $\frac{\gamma-K_{\sigma, z}}{\kappa+K_{\sigma, z}} \leq \frac{\gamma}{\kappa \vee K_{\sigma, z}} \leq 1$, and $1-\frac{\gamma+\kappa}{L_{\sigma, z}+\kappa}<1$ because the second term is positive. In particular, if $K_{\xi, x}=0$ we have the strict inequality $\frac{\gamma-K_{\sigma, z}}{\kappa+K_{\sigma, z}} \leq \frac{\gamma}{\kappa \vee K_{\sigma, z}}<1$ by the choice of $\kappa$ and $\gamma$. We conclude that $L_{\hat{\sigma}, z}<1$ if $K_{\xi, x}=0$, and $L_{\hat{\sigma}, z} \leq 1$ if $K_{\xi, x}>0$.

We obtain the contraction condition $L_{\hat{\xi}, x}<L_{\hat{\sigma}, z}^{-1}$ by the calculations above and therefore $(\hat{\xi}, \hat{\mu}, \hat{\sigma}, \hat{f})$ fulfill SLC. Finally, the second part of the Proposition follows by Theorem 3.4 .

Proof of Theorem 4.3. Let $t \in I_{\max }, x \in \mathbb{R}$ and the mapping $x \mapsto(\kappa \operatorname{Id}+\gamma \hat{u}(t, \cdot))(x)$ be bijective. Define $\hat{x}:=(\kappa \operatorname{Id}+\gamma \hat{u}(t, \cdot))^{-1}((\kappa+\gamma) x)$. According to Proposition 4.2 and Theorem 3.4 there exists a solution $(\hat{X}, \hat{Y}, \hat{Z})$ to FBSDE $(4.1)$ on $[t, T]$ with the $\mathcal{F}_{t}$-measurable initial condition $\hat{x}$. If we now apply Lemma 4.6 we obtain that there exists a solution $(X, Y, Z)$ to FBSDE $(1.1)$ on $[t, T]$ with initial condition

$$
X_{t}=\frac{\kappa \hat{X}_{t}+\gamma \hat{Y}_{t}}{\kappa+\gamma}=\frac{1}{\kappa+\gamma}(\kappa \operatorname{Id}+\gamma \hat{u}(t, \cdot))(\hat{x})=x \in \mathbb{R} .
$$


It remains to show that $u$ is a decoupling field for $(\xi, \mu, \sigma, f)$. To this end, we verify Definition 3.1. Note that in contrast to the first part of the proof we consider the FBSDE (1.1) on a time interval $\left[t_{1}, t_{2}\right] \subseteq[t, T]$ for some fixed $t \in I_{\max }$ and with an $\mathcal{F}_{t_{1}}$-measurable initial condition $X_{t_{1}}: \Omega \rightarrow \mathbb{R}$. Since $\hat{u}$ is a decoupling field for $(\hat{\xi}, \hat{\mu}, \hat{\sigma}, \hat{f})$ there are also processes $(\hat{X}, \hat{Y}, \hat{Z})$ that satisfy $(3.1)$ with initial condition $\hat{X}_{t_{1}}:=\left(\kappa \operatorname{Id}+\gamma \hat{u}\left(t_{1}, \cdot\right)\right)^{-1}\left((\kappa+\gamma) X_{t_{1}}\right)$. If we define $(X, Y, Z)$ as in Lemma 4.6 we obtain by calculations that are analogous to the proof of Lemma 4.6 that

$$
\begin{aligned}
& X_{s}=X_{t_{1}}+\int_{t_{1}}^{s} \mu\left(r, X_{r}, Y_{r}, Z_{r}\right) d r+\int_{t_{1}}^{s} \sigma\left(r, X_{r}, Y_{r}, Z_{r}\right) d W_{r}, \\
& Y_{s}=Y_{t_{2}}-\int_{s}^{t_{2}} f\left(r, X_{r}, Y_{r}, Z_{r}\right) d r-\int_{s}^{t_{2}} Z_{r} d W_{r}, \text { a.s., } s \in\left[t_{1}, t_{2}\right] .
\end{aligned}
$$

To verify the decoupling condition, note that $(\kappa \operatorname{Id}+\gamma \hat{u}(s, \cdot))\left(\hat{X}_{s}\right)=(\kappa+\gamma) X_{s}$ and thus

$$
\begin{aligned}
u\left(s, X_{s}\right) & =\frac{1}{\kappa+\gamma}\left((\operatorname{Id}-\hat{u}(s, \cdot)) \circ(\kappa \operatorname{Id}+\gamma \hat{u}(s, \cdot))^{-1}\right)\left((\kappa+\gamma) X_{s}\right) \\
& =\frac{1}{\kappa+\gamma}(\operatorname{Id}-\hat{u}(s, \cdot))\left(\hat{X}_{s}\right)=\frac{\hat{X}_{s}-\hat{Y}_{s}}{\kappa+\gamma}=Y_{s}, \text { a.s., } s \in\left[t_{1}, t_{2}\right] .
\end{aligned}
$$

Hence, the property $(3.1)$ is fulfilled. Finally, we prove that $u(T, \cdot)=\xi$. If $\gamma=0$ we have

$$
u(T, x)=\frac{1}{\kappa}(\operatorname{Id}-\hat{u}(T, \cdot))(x)=\frac{1}{\kappa}(\operatorname{Id}-\hat{\xi})(x)=\xi(x), x \in \mathbb{R} .
$$

If $\gamma>0$ we observe by (4.3) that for all $x \in \mathbb{R}$

$$
(\kappa \operatorname{Id}+\gamma \hat{\xi})(x)=\left(\kappa \operatorname{Id}+(\kappa+\gamma)(\operatorname{Id}+\gamma \xi)^{-1}-\kappa \operatorname{Id}\right)(x)=(\kappa+\gamma)(\operatorname{Id}+\gamma \xi)^{-1}(x),
$$

and thus, $(\kappa \operatorname{Id}+\gamma \hat{\xi})^{-1}(x)=(\operatorname{Id}+\gamma \xi)\left(\frac{x}{\kappa+\gamma}\right), x \in \mathbb{R}$. Therefore, it follows

$$
\begin{aligned}
u(T, x) & =\frac{1}{\kappa+\gamma}\left((\operatorname{Id}-\hat{\xi}) \circ(\kappa \operatorname{Id}+\gamma \hat{\xi})^{-1}\right)((\kappa+\gamma) x) \\
& =\frac{1}{\kappa+\gamma}((\operatorname{Id}-\hat{\xi}) \circ(\operatorname{Id}+\gamma \xi))(x) \\
& =\frac{1}{\kappa+\gamma}\left(\left(\frac{\kappa+\gamma}{\gamma} \operatorname{Id}-\frac{\kappa+\gamma}{\gamma}(\operatorname{Id}+\gamma \xi)^{-1}\right) \circ(\operatorname{Id}+\gamma \xi)\right)(x)=\xi(x), x \in \mathbb{R}
\end{aligned}
$$

We conclude that $u$ is a decoupling field for $(\xi, \mu, \sigma, f)$.

Proof of Corollary 4.4. Let $x \in \mathbb{R}$. We are in the setting of Theorem 4.3 with $t=0$ and therefore we can argue as in the previous proof. In fact, the initial value $\hat{x}=(\kappa \operatorname{Id}+\gamma \hat{u}(0, \cdot))^{-1}((\kappa+\gamma) x)$ of the transformed FBSDE (4.1) is constant a.s. since it is $\mathcal{F}_{0}$-measurable. Therefore, we obtain additionally that the solution $(\hat{X}, \hat{Y}, \hat{Z})$ to FBSDE (4.1) has the property (3.3) by Theorem 3.4. Consequently, also the solution $(X, Y, Z)$ to FBSDE (1.1) satisfies (3.3), because $X$ and $Y$ are 
linear combinations of $\hat{X}$ and $\hat{Y}$, and for some constant $C \geq 0$ we have

$$
\begin{aligned}
\mathbb{E}\left[\int_{0}^{T}\left|Z_{s}\right|^{2} d s\right] & =\mathbb{E}\left[\int_{0}^{T}\left|\hat{\varphi}\left(s, \hat{X}_{s}, \hat{Y}_{s}, \hat{Z}_{s}\right)\right|^{2} d s\right] \\
& =\mathbb{E}\left[\int_{0}^{T}\left|\hat{\varphi}\left(s, \hat{X}_{s}, \hat{Y}_{s}, \hat{Z}_{s}\right)-\hat{\varphi}(s, 0,0,(\sigma(s, 0,0, \cdot)-\kappa \operatorname{Id})(0))\right|^{2} d s\right] \\
& \leq \mathbb{E}\left[\int_{0}^{T} C\left(\left|\hat{X}_{s}\right|^{2}+\left|\hat{Y}_{s}\right|^{2}+\left|\hat{Z}_{s}\right|^{2}+\|\sigma(\cdot, \cdot, 0,0,0)\|_{\infty}^{2}\right) d s\right]<\infty,
\end{aligned}
$$

due to $(\hat{X}, \hat{Y}, \hat{Z})$ having the property (3.3), the property (A3) and the Lipschitz continuity of $\hat{\varphi}$ (see Lemma 4.7). Moreover, $(X, Y, Z)$ is the unique solution to FBSDE (1.1) satisfying (3.3): Let $\left(X^{(2)}, Y^{(2)}, Z^{(2)}\right)$ be another solution to (1.1) on $[0, T]$ fulfilling (3.3). Then $\left(\hat{X}^{(2)}, \hat{Y}^{(2)}, \hat{Z}^{(2)}\right)$, defined as

$$
\hat{X}_{s}^{(2)}:=X_{s}^{(2)}+\gamma Y_{s}^{(2)}, \hat{Y}_{s}^{(2)}:=X_{s}^{(2)}-\kappa Y_{s}^{(2)}, \hat{Z}_{s}^{(2)}:=\sigma\left(s, X_{s}^{(2)}, Y_{s}^{(2)}, Z_{s}^{(2)}\right)-\kappa Z_{s}^{(2)}
$$

for $s \in[0, T]$, solves (4.1) on $[0, T]$ and satisfies (3.3). But solutions to (4.1) with (3.3) are unique according to Theorem 3.4 and thus $(\hat{X}, \hat{Y}, \hat{Z})=\left(\hat{X}^{(2)}, \hat{Y}^{(2)}, \hat{Z}^{(2)}\right)$. Consequently, we also have $(X, Y, Z)=\left(X^{(2)}, Y^{(2)}, Z^{(2)}\right)$.

\section{$5 \quad$ Examples}

In this section we present several applications of linear transformations combined with the method of decoupling fields. In all these applications we proceed as follows: Assume we have an FBSDE satisfying (A1)-(A5), but not the contraction condition (1.2). Then we choose $\kappa$ and $\gamma$ such that the auxiliary FBSDE (4.1) satisfies SLC. Next, we verify whether (4.1) possesses a global solution $(\hat{X}, \hat{Y}, \hat{Z})$ with a suitable technique (the method of decoupling fields in our case). If it does, then the triplet $(X, Y, Z)$, defined as in Lemma 4.6, solves the original FBSDE if the bijectivity condition in Corollary 4.4 is satisfied.

\subsection{A linear example with an explicit solution}

We modify Example 1.1 such that it satisfies our assumptions and show that it is possible to determine a solution via a transformation. Consider for some initial value $x \in \mathbb{R}$ the FSBDE

$$
\begin{aligned}
& X_{s}=x+\int_{0}^{s}\left(\sigma_{0}-Z_{r}\right) d W_{r}, \\
& Y_{s}=X_{T}-\int_{s}^{T} Z_{r} d W_{r}, s \in[0, T],
\end{aligned}
$$

where $\sigma_{0} \in \mathbb{R}$.

Proposition 5.1. For every intial value $x \in \mathbb{R}$ there exists a solution $(X, Y, Z)$ to the FBSDE (5.1) on $[0, T]$. 
Proof. Note that $\mu, f \equiv 0, \sigma(z)=\sigma_{0}-z$ and $\xi(x)=x$. Therefore, $L_{\sigma, z}=L_{\xi, x}=$ $1, K_{\sigma, z}=1$ and $\sigma$ is strictly decreasing, while $\xi$ is strictly increasing. If we choose $\kappa:=1$ and $\gamma:=0$, i.e. the transformation with

$$
\left(\begin{array}{cc}
1 & 0 \\
1 & -1
\end{array}\right)
$$

we observe that $\hat{\xi} \equiv 0$ and the auxiliary FBSDE takes the form

$$
\begin{aligned}
& \hat{X}_{s}=x+\frac{1}{2} \int_{0}^{s}\left(\sigma_{0}+\hat{Z}_{r}\right) d W_{r}, \\
& \hat{Y}_{s}=-\int_{s}^{T} \hat{Z}_{r} d W_{r}, s \in[0, T],
\end{aligned}
$$

for some initial value $x \in \mathbb{R}$. It is obvious that $0=L_{\hat{\xi}, x}<L_{\hat{\sigma}, z}^{-1}=2$ and that $(\hat{X}, \hat{Y}, \hat{Z})$, defined by $\hat{X}_{s}:=x+\frac{1}{2} \sigma_{0} W_{s}, \hat{Y}_{s}, \hat{Z}_{s}:=0, s \in[0, T]$, solves the FBSDE (5.2) on $[0, T]$. Then the backward transformation in Lemma 4.6 implies that $(X, Y, Z)$, given by $X_{s}:=\hat{X}_{s}, Y_{s}:=\hat{X}_{s}, Z_{s}:=\frac{1}{2} \sigma_{0}, s \in[0, T]$, solves the FBSDE (5.1) on $[0, T]$ with initial condition $x$. It is also straightforward to verify that this is indeed true.

\subsection{A transformation bounding the gradient process}

We consider the FBSDE

$$
\begin{aligned}
& X_{s}=x+\int_{0}^{s}\left(2 X_{r}+\frac{1}{2} Y_{r}\right) d r-\int_{0}^{s}\left(X_{r}+Y_{r}+Z_{r}\right) d W_{r}, \\
& Y_{s}=X_{T}-\int_{s}^{T} Z_{r} d W_{r}, s \in[0, T] .
\end{aligned}
$$

Our goal is to transform the FBSDE (5.3) into an FBSDE satisfying SLC. Then we apply the method of decoupling fields to show that there is a solution on $[0, T]$ to the auxiliary FBSDE. Finally, by using Corollary 4.4 we fully characterize the solvability of (5.3) in dependence of the time to maturity $T$.

Proposition 5.2. If $T \neq \frac{\ln (5)}{2}$, then for every initial value $x \in \mathbb{R}$ there exists a unique solution $(X, Y, Z)$ to FBSDE (5.3) on $[0, T]$ satisfying (3.3) with $t=0$.

If $T=\frac{\ln (5)}{2}$, then such solutions exist for $x=0$ only and in this case there are infinitely many solutions.

Proof. Note that $\xi(x)=x, \mu(x, y)=2 x+\frac{1}{2} y, \sigma(x, y, z)=-x-y-z, f \equiv 0$ and $L_{\sigma, z}=L_{\xi, x}=1, K_{\sigma, z}=1$. Consequently, the parameters do not satisfy SLC, but they fulfill our assumptions (A1)-(A5). Since $K_{\sigma, z}>0$ we can choose $\kappa:=0$ and $\gamma:=\frac{1}{2}$ (see Remark 4.1), i.e. we consider the transformation with

$$
\left(\begin{array}{ll}
1 & \frac{1}{2} \\
1 & 0
\end{array}\right)
$$


Then the auxiliary FBSDE takes the form

$$
\begin{aligned}
& \hat{X}_{s}=\hat{x}+\int_{0}^{s}\left(\hat{X}_{r}+\hat{Y}_{r}\right) d r+\int_{0}^{s}\left(-\hat{X}_{r}+\frac{1}{2} \hat{Y}_{r}+\frac{1}{2} \hat{Z}_{r}\right) d W_{r}, \\
& \hat{Y}_{s}=\frac{2}{3} \hat{X}_{T}-\int_{s}^{T}\left(\hat{X}_{r}+\hat{Y}_{r}\right) d r-\int_{s}^{T} \hat{Z}_{r} d W_{r}, s \in[0, T] .
\end{aligned}
$$

Now, the new parameters satisfy SLC because $L_{\hat{\xi}, x}=\frac{2}{3}<2=L_{\hat{\sigma}, z}^{-1}$. According to Proposition 4.2 there exists a weakly regular decoupling field $\hat{u}$ and a maximal interval $I_{\max } \subseteq[0, T]$ for $(\hat{\xi}, \hat{\mu}, \hat{\sigma}, \hat{f})$ such that for all $t \in I_{\max }$ the FBSDE (5.4) has a solution on $[t, T]$ for every initial value $x \in \mathbb{R}$. We prove that there is also a solution on $[0, T]$ with the help of the method of decoupling fields. To this end, assume that $I_{\max }=\left(t_{\min }, T\right]$ for some $t_{\min } \in[0, T]$ and fix some $t_{0} \in I_{\max }$. There exists a solution $(\hat{X}, \hat{Y}, \hat{Z})$ of $(5.4)$ on $\left[t_{0}, T\right]$ according to Theorem 3.4. We aim at showing that $L_{\hat{u}(t, \cdot), x}$ can be bounded away from $L_{\hat{\sigma}, z}^{-1}=2$ independently of $t$ by studying the dynamics of the so-called gradient process $V_{t}=\partial_{x} \hat{u}\left(t, \hat{X}_{t}\right)$. Theorem 4.1 in [6] implies that $V$ satisfies the BSDE

$$
V_{s}=\frac{2}{3}-\int_{s}^{T} \varphi\left(r, V_{r}, \tilde{Z}_{r}\right) d r-\int_{s}^{T} \tilde{Z}_{r} d W_{r}, s \in\left[t_{0}, T\right],
$$

where $\varphi(s, v, z):=(1-v)(1+v)-z\left(-1+\frac{1}{2} v+\frac{1}{2} \frac{-v+\frac{1}{2} v^{2}+z}{1-\frac{1}{2} v}\right)$. We observe that

$$
\begin{aligned}
\left|-1+\frac{1}{2} V_{s}+\frac{1}{2} \frac{-V_{s}+\frac{1}{2} V_{s}^{2}+\tilde{Z}_{s}}{1-\frac{1}{2} V_{s}}\right| & \leq 1+\frac{C}{2}+\frac{C}{2-C}+\frac{1}{2} \frac{C^{2}}{2-C}+\frac{\left|\tilde{Z}_{s}\right|}{2-C} \\
& \leq \tilde{C}\left(1+\left|\tilde{Z}_{s}\right|\right)
\end{aligned}
$$

for some constant $\tilde{C}>0$, because $V$ is bounded by a constant $C<2=L_{\hat{\sigma}, z}^{-1}$ on $\left[t_{0}, T\right]$ due to the weak regularity of $\hat{u}$. In particular, the generator $\varphi$ has at most quadratic growth in $\tilde{Z}$. Therefore, $\tilde{Z}$ is a $B M O(\mathbb{P})$-process (consult e.g. [2]) and, thus, there is a probability measure $\mathbb{Q} \sim \mathbb{P}$ such that

$$
V_{s}=\frac{2}{3}-\int_{s}^{T}\left(1-V_{r}\right)\left(1+V_{r}\right) d r-\int_{s}^{T} \tilde{Z}_{r} d W_{r}^{\mathbb{Q}}, s \in\left[t_{0}, T\right],
$$

where $W^{\mathbb{Q}}$ is a Brownian motion with drift under $\mathbb{P}$ and a Brownian motion under $\mathbb{Q}$. It is straightforward to verify that $(5.5)$ is also satisfied by the pair of processes $(\bar{V}, \bar{Z})$, where $\bar{Z}=0$ and $\bar{V}$ is given by

$$
\bar{V}_{s}=\frac{5 e^{2 s}-e^{2 T}}{5 e^{2 s}+e^{2 T}}=\frac{5-e^{2(T-s)}}{5+e^{2(T-s)}}, s \in\left[t_{0}, T\right]
$$

By uniqueness of solutions to BSDEs we have $(V, \tilde{Z})=(\bar{V}, \bar{Z})$. This implies

$$
V_{s} \leq \frac{5-e^{2(T-T)}}{5+e^{2(T-T)}}=\frac{2}{3} \quad \text { and } \quad V_{s} \geq \frac{5-e^{2 T}}{5+e^{2 T}}>-1, \quad s \in\left[t_{0}, T\right] .
$$


Considering $L_{\hat{\sigma}, z}^{-1}=2$, Theorem 3.4 yields that only $I_{\max }=[0, T]$ is possible. Therefore, we obtain solutions to (5.4) for arbitrary $T>0$ and $\hat{x} \in \mathbb{R}$.

In order to apply Theorem 4.3 and Corollary 4.4 we need bijectivity of $\hat{u}(0, \cdot)$. Note that $\partial_{x} \hat{u}(0, \cdot)$ is known to be constant $\frac{5-e^{2 T}}{5+e^{2 T}}$. Thus, $\hat{u}(0, \cdot)$ is a linear function. It is indeed bijective if $T \neq \frac{\ln (5)}{2}$. In this case we have solutions to (5.3) which are unique (under $(3.3)$ ) because of the one-to-one correspondence between $(\hat{X}, \hat{Y}, \hat{Z})$ and $(X, Y, Z)$ solving (5.4) and (5.3), respectively.

It remains to consider the case $T=\frac{\ln (5)}{2}$ : Assume that we have a solution $(X, Y, Z)$ to $(5.3)$ on $[0, T]$ s.t. $(3.3)$ for $t=0$ is satisfied. Then the associated $(\hat{X}, \hat{Y}, \hat{Z})$, obtained via a linear transformation, satisfies (5.4). In accordance with Theorem 3.4 the decoupling condition holds, in particular $x=X_{0}=\hat{Y}_{0}=\hat{u}(0, \hat{x})=\hat{u}\left(0, x+\frac{1}{2} Y_{0}\right)$. We can determine $\hat{u}(0, \hat{x})$ explicitly, since $\hat{u}(0, \cdot)$ is a constant function for $T=\frac{\ln (5)}{2}$ : This constant is equal zero, since the zero triplet for $(\hat{X}, \hat{Y}, \hat{Z})$ solves (5.4). Thus, we obtain that $x=0$ must hold if (5.3) has a solution satisfying (3.3) with $t=0$. If, however, $x=0$ is satisfied then we have infinitely many solutions: One can construct solutions by choosing an arbitrary $\hat{x}$ and obtaining the associate $(\hat{X}, \hat{Y}, \hat{Z})$, which then yields a suitable $(X, Y, Z)$ via a linear transformation. Since $\hat{x}=\frac{1}{2} Y_{0}$ and all $\hat{x}$ are different we have infinitely many different solutions to (5.3).

Remark 5.3. Note that also other results in the existing literature guarantee global solvability of the auxiliary FBSDE, like e.g. Theorem 7.4 in [11]. However, since the original FBSDE (5.3) does not have a solution for all $T>0$ and all initial values $x \in \mathbb{R}$ one cannot apply general existence results for FBSDEs to the original FBSDE (5.3). Our approach allows a full characterization of the solvability of (5.3) by studying the decoupling field of the auxiliary FBSDE (5.4).

\subsection{The adjoint equation in a diffusion control problem}

In this section we present diffusion control problems which are studied in [1] and [16]. In particular, we study the controlled diffusion process $X^{x, \alpha}$ with dynamics

$$
d X_{s}^{x, \alpha}=\left(b_{s}+B_{s} X_{s}^{x, \alpha}\right) d s+\alpha_{s} d W_{s}, X_{0}^{x, \alpha}=x \in \mathbb{R} .
$$

The control problem consists of minimizing the cost functional

$$
\mathbb{E}\left[\int_{0}^{T} f\left(s, \alpha_{s}\right) d s+g\left(X_{T}^{x, \alpha}\right)\right]
$$

over all progressively measurable and $L^{2}$-integrable controls $\alpha$ taking values in a given set $A \subseteq \mathbb{R}$, specified later. We call such controls admissible and denote the set of all admissible controls by $\mathcal{A}$. Furthermore, we assume the following:

(C1) $f: \Omega \times[0, T] \times A \rightarrow \mathbb{R}$ is a measurable function such that the mapping $(\omega, t) \mapsto f(\omega, t, a)$ is progressively measurable for all $a \in A$. In addition, $f(t, \cdot)$ is convex and twice continuously differentiable with uniformly bounded second derivative and locally bounded first derivatives (locally in $a$ ). In particular, $f_{a a}$ is bounded away from zero by a constant $\delta_{l}>0$ and bounded from above by a constant $\delta_{u}$. 
(C2) $g: \Omega \times \mathbb{R} \rightarrow \mathbb{R}$ is a measurable, convex and twice continuously differentiable function with bounded second derivative and locally bounded first derivative. Moreover, $g^{\prime \prime}$ is bounded away from zero by $\delta_{l}$ and bounded from above by $\delta_{u}$.

(C3) $b, B: \Omega \times[0, T] \rightarrow \mathbb{R}$ are progressively measurable and bounded processes.

Remark 5.4. The conditions (C1)-(C3) together with the assumptions on the admissible control processes ensure that (5.6) has a unique strong solution for any initial value $x \in \mathbb{R}$.

Control problems of this type arise in situations where one can control a state's fluctuation intensity but not its drift, and where one aims at steering the state into a target area. To give an explicit example, $X^{x, \alpha}$ may describe the position of a particle in a medium with temperature $\alpha$. By heating or cooling the medium the particle's fluctuations increase or decrease, respectively. The function $f$ reflects the costs involved by any temperature change. Diffusion control problems arise also in portfolio optimization. In this context $X^{x, \alpha}$ can be interpreted as a portfolio value process with volatility $\alpha$. A reduction of the portfolio's volatility involves hedging costs $f$. The function $-g$ can be taken to be a utility function. The optimal control of diffusion coefficients appear also in other fields of applications, see e.g. [13] for examples arising in biology.

In both works, [1] and [16], the transformation method described in this paper is used with a particular choice of the parameters $\kappa$ and $\gamma$ to show that there exists a solution to the adjoint FBSDE derived from Pontryagin's maximum principle. This allows to prove the existence of an optimal control. In [1] the case $A=\mathbb{R}$ is studied, i.e. the case of unbounded control processes. Here also the assumptions $(\mathrm{C} 1)$ and $(\mathrm{C} 2)$ are relaxed: $f$ is allowed to depend on the current state $X_{t}^{x, \alpha}$ and $f_{a a}(t, \cdot), g^{\prime \prime}$ are not necessarily bounded away from zero. An application of a linear transformation with $\kappa=0$ and $\gamma=\delta_{u}^{-1}$ together with the method of decoupling fields allows to verify the existence of an optimal control in this setting. We refer to [1] for more details.

We focus here on the case where $A=[l, r]$ is a compact non-empty interval, which is studied in chapter 3 of the thesis [16]. All details can be found there. This slightly different setting requires a completely different transformation because the adjoint FBSDEs of both control problems differ. In particular, the diffusion coefficient $\sigma$ in the compact interval case is not invertible in $z$ unlike in [1], which implies that $\kappa$ has to be positive.

As mentioned above, we aim at finding an optimal control $\hat{\alpha} \in \mathcal{A}$ such that

$$
\mathbb{E}\left[\int_{0}^{T} f\left(s, \hat{\alpha}_{s}\right) d s+g\left(X_{T}^{x, \hat{\alpha}}\right)\right]=\inf _{\alpha \in \mathcal{A}} \mathbb{E}\left[\int_{0}^{T} f\left(s, \alpha_{s}\right) d s+g\left(X_{T}^{x, \alpha}\right)\right] .
$$

We choose a probabilistic approach via Pontryagin's maximum principle to solve this problem because of the non-Markovian framework. Note that the Hamiltonian of the control problem is minimized by the function $a_{*}(t,-z)$, where

$$
a_{*}(t, z):= \begin{cases}l & , z<f_{a}(t, l), \\ f_{a}^{-1}(t, z) & , z \in\left[f_{a}(t, l), f_{a}(t, r)\right], \quad(t, z) \in[0, T] \times \mathbb{R} . \\ r & , z>f_{a}(t, r),\end{cases}
$$


Pontryagin's maximum principle implies that $\hat{\alpha}$, given by

$$
\hat{\alpha}_{s}:=a_{*}\left(s,-Z_{s}\right), s \in[0, T],
$$

is an optimal control of (5.7), if there exists a solution $(X, Y, Z)$ to the following adjoint FBSDE:

$$
\begin{aligned}
& X_{t}=x+\int_{0}^{t}\left(b_{s}+B_{s} X_{s}\right) d s+\int_{0}^{t} a_{*}\left(s,-Z_{s}\right) d W_{s}, \\
& Y_{t}=g^{\prime}\left(X_{T}\right)+\int_{t}^{T} B_{s} Y_{s} d s-\int_{t}^{T} Z_{s} d W_{s}, t \in[0, T] .
\end{aligned}
$$

The FBSDE (5.9) has the parameters

$$
\begin{aligned}
\mu(s, x) & :=b_{s}+B_{s} x, \\
\sigma(s, z) & :=a_{*}(s,-z), \\
f(s, y) & :=B_{s} y, \\
\xi(x) & :=g^{\prime}(x),
\end{aligned}
$$

for $(s, x, y, z) \in[0, T] \times \mathbb{R} \times \mathbb{R} \times \mathbb{R}$. We observe that the conditions (A1)-(A5) are satisfied, $L_{\sigma, z} \leq \frac{1}{\delta_{l}}$ and $L_{\xi, x} \leq \delta_{u}$. Notice that the contraction condition (1.2) might not hold true. Linear transformations, however, allow further examination of the solvability of (5.9). We obtain the following existence result:

Proposition 5.5. The FBSDE (5.9) has a unique solution $(X, Y, Z)$ on $[0, T]$ for any initial value $x \in \mathbb{R}$. Moreover, the control $\hat{\alpha} \in \mathcal{A}$, given by (5.8), is optimal for the minimization problem (5.7).

Proof. We want to transform the FBSDE (5.9) into an auxiliary FBSDE satisfying SLC. Since $K_{\sigma, z}=0, K_{\xi, x}=\delta_{l}$ we can choose $\gamma:=\left(2 \delta_{u}\right)^{-1}$ and $\kappa:=\gamma$, and consider the linear transformation with

$$
\left(\begin{array}{cc}
1 & \gamma \\
1 & -\gamma
\end{array}\right)
$$

The choice of $\kappa$ and $\gamma$ might appear arbitrary because there are, of course, other choices which transform the FBSDE (5.9) into an FBSDE satisfying the contraction condition. The advantage of this choice is that one can calculate a uniform bound for the gradient process (see section 3.4 in [16]), which can fail with other choices. That makes this selection adequate.

The transformation (5.10) yields the auxiliary FBSDE

$$
\begin{aligned}
& \hat{X}_{t}=x+\int_{0}^{t}\left(b_{s}+B_{s} \hat{Y}_{s}\right) d s+\int_{0}^{t}\left(\hat{Z}_{s}-2 \gamma \tilde{a}_{*}^{-1}\left(s, \hat{Z}_{s}\right)\right) d W_{s}, \\
& \hat{Y}_{t}=\hat{\xi}\left(X_{T}\right)-\int_{t}^{T}\left(b_{s}+B_{s} \hat{X}_{s}\right) d s-\int_{t}^{T} \hat{Z}_{s} d W_{s}, t \in[0, T]
\end{aligned}
$$


with initial value $x \in \mathbb{R}$, where

$$
\begin{aligned}
\hat{\xi}(\omega, x) & :=\left(\left(\operatorname{Id}-\gamma g^{\prime}(\omega, \cdot)\right) \circ\left(\operatorname{Id}+\gamma g^{\prime}(\omega, \cdot)\right)^{-1}\right)(x), \\
\tilde{a}_{*}^{-1}(\omega, t, z) & :=\left(a_{*}(\omega, t, \cdot)+\gamma \operatorname{Id}\right)^{-1}(z) \\
& = \begin{cases}\frac{1}{\gamma}(z-l) & , z<l+\gamma f_{a}(\omega, t, l), \\
\left(f_{a}^{-1}(\omega, t, \cdot)+\gamma \operatorname{Id}\right)^{-1}(z) & , z \in\left[l+\gamma f_{a}(\omega, t, l), r+\gamma f_{a}(\omega, t, r)\right], \\
\frac{1}{\gamma}(z-r) & , z>r+\gamma f_{a}(\omega, t, r),\end{cases}
\end{aligned}
$$

for all $(\omega, t, x, z) \in \Omega \times[0, T] \times \mathbb{R} \times \mathbb{R}$. From Proposition 4.2 we know that the parameters of (5.9) satisfy SLC and, in particular, the contraction condition. One can even explicitly calculate bounds for the Lipschitz constants $L_{\hat{\sigma}, z}$ and $L_{\hat{\xi}, x}$ similar to the proof of Proposition 4.2. One obtains that $L_{\hat{\xi}, x} \leq \frac{1}{1+\gamma \delta_{l}}<1=L_{\hat{\sigma}, z}^{-1}$ (see Proposition 3.6 in $[16$, p. 41]).

Now we can apply the method of decoupling fields in order to show that there exists a solution $(\hat{X}, \hat{Y}, \hat{Z})$ to the auxiliary FBSDE (5.11) on $[0, T]$. Since the parameters of (5.11) satisfy SLC, Theorem 3.4 implies the existence of a weakly regular decoupling field $\hat{u}$ on $I_{\max }$, which has either the form $[0, T]$ or $\left(t_{\min }, T\right]$. The method of decoupling fields consists in verifying that the case $I_{\max }=\left(t_{\min }, T\right]$ and thus $\lim _{t \downarrow t_{\min }} L_{\hat{u}(t, \cdot), x}=L_{\hat{\sigma}, z}^{-1}$ cannot hold true. To that end, we study the gradient of $\hat{u}(t, \cdot)$ with the help of the so-called gradient process and bound it away from $L_{\hat{\sigma}, z}^{-1}$ independently of $t$.

Assume $I_{\max }=\left(t_{\min }, T\right]$ for some $t_{\min } \in[0, T)$. Fix an initial time point $t_{0} \in I_{\max }$ for the auxiliary FBSDE (5.11). Let $(\hat{X}, \hat{Y}, \hat{Z})$ be a solution to $(5.11)$ on $\left[t_{0}, T\right]$ with initial value $x \in \mathbb{R}$ and define the gradient process $V$ by $V_{t}:=\hat{u}_{x}\left(t, \hat{X}_{t}\right), t \in\left[t_{0}, T\right]$. Note that we have in particular $V_{t_{0}}=\hat{u}_{x}\left(t_{0}, x\right)$. If we differentiate the decoupling condition $\hat{Y}_{t}=\hat{u}\left(t, \hat{X}_{t}\right)$ w.r.t. the initial value $x$ we obtain by the chain rule that $\partial_{x} \hat{Y}_{t}=V_{t} \partial_{x} \hat{X}_{t}, t \in\left[t_{0}, T\right]$, and hence

$$
V_{t}=\frac{\partial_{x} \hat{Y}_{t}}{\partial_{x} \hat{X}_{t}}, t \in\left[t_{0}, \tau\right)
$$

where $\tau:=\inf \left\{t \in\left[t_{0}, T\right]: \partial_{x} \hat{X}_{t}=0\right\} \wedge T$. Indeed, one can differentiate the processes $\hat{X}, \hat{Y}, \hat{Z}$ w.r.t. the initial value at least in a weak sense, see e.g. Theorem 2.5.11 in [7] or Theorem 3.4 in [1]. Moreover, one obtains the dynamics of the processes $\partial_{x} \hat{X}$ and $\partial_{x} \hat{Y}$ by differentiation of the right hand sides of (5.11) using the chain rule and interchanging differentiation and integration. That particularly implies that $V$ is an Itô process (after possible adaptation on a subset of $\Omega \times\left[t_{0}, T\right]$ with measure zero, see Lemma 3.12 in [16]), and thus has the representation

$$
V_{t}=u_{x}\left(t_{0}, x\right)+\int_{t_{0}}^{t} \eta_{s} d s+\int_{t_{0}}^{t} \tilde{Z}_{s} d s, t \in\left[t_{0}, \tau\right),
$$

for some processes $\eta$ and $\tilde{Z}$. We can determine these processes by applying Itô's formula to the identity $\partial_{x} \hat{Y}_{t}=V_{t} \partial_{x} \hat{X}_{t}$. Moreover, we can show that $\tau=T$ a.s. and thus we can write the gradient process $V$ in the backward form and obtain that 
$(V, \tilde{Z})$ solves the following BSDE:

$$
V_{t}=\hat{\xi}^{\prime}\left(\hat{X}_{T}\right)-\int_{t}^{T}\left(B_{s}\left(1-V_{s}\right)\left(1+V_{s}\right)-\frac{\Sigma_{s}}{1-\Sigma_{s} V_{s}} \tilde{Z}_{s}^{2}\right) d s-\int_{t}^{T} \tilde{Z}_{s} d W_{s},
$$

on $\left[t_{0}, T\right]$. Here $\Sigma$ denotes a process, which appears by differentiation of $\hat{\sigma}\left(t, \hat{Z}_{t}\right)$ w.r.t. the initial value. We refer again to Lemma 3.12 in [16] for details.

The BSDE representation (5.12) for $V$ can be simplified: Since $\tilde{Z}$ is a $B M O(\mathbb{P})$ process there exists an equivalent probability measure $Q$ and a Brownian motion $W^{Q}$ under $Q$ such that (5.12) takes the form

$$
V_{t}=\hat{\xi}^{\prime}\left(\hat{X}_{T}\right)-\int_{t}^{T} B_{s}\left(1-V_{s}\right)\left(1+V_{s}\right) d s-\int_{t}^{T} \tilde{Z}_{s} d W_{s}^{Q}, t \in\left[t_{0}, T\right],
$$

(see Lemma 3.13 in [16] for details). Standard comparison arguments for BSDEs now imply that

$$
\left|V_{t}\right| \leq 1-\left(1-\frac{1}{1+\gamma \delta_{l}}\right) \exp \left(-2\|B\|_{\infty} T\right)=: q<1, t \in\left[t_{0}, T\right], \text { a.s. }
$$

We emphasize that the arguments above are only true for almost all $x \in \mathbb{R}$, which we have not mentioned so far. Nevertheless, this is sufficient since we obtain that $\left|\hat{u}_{x}\left(t_{0}, x\right)\right| \leq q<1$ a.e., and therefore

$$
\lim _{t \downarrow t_{\min }} L_{\hat{u}(t, \cdot), x} \leq q<1 \leq L_{\hat{\sigma}, z}^{-1} .
$$

Theorem 3.4 implies that only $I_{\max }=[0, T]$ is possible. Hence, for any initial value $x \in \mathbb{R}$ there exists a unique solution to the FBSDE (5.11).

Finally, it remains to show global solvability of our original FBSDE (5.9). To this end, note that the mapping $\gamma(\operatorname{Id}+\hat{u}(0, \cdot))$ is invertible since $L_{\hat{u}, x} \leq q<1$. Corollary 4.4 implies that also FBSDE (5.9) has a solution $(X, Y, Z)$ on $[0, T]$ and Pontryagin's maximum principle implies that $\hat{\alpha}$, given by $\hat{\alpha}_{s}:=a_{*}\left(s,-Z_{s}\right), s \in[0, T]$, is an optimal control.

Remark 5.6. For the adjoint FBSDE (5.9), as well as the auxiliary FBSDE (5.11), the literature does not offer existence results for global solutions to the best of our knowledge. The application of linear transformations and the method of decoupling fields, however, admits to treat this class of equations. In some cases one can even show global existence of solution, as in the above setting.

\section{References}

[1] S. Ankirchner and A. Fromm. "Optimal Control of Diffusion Coefficients via Decoupling Fields". In: SIAM Journal on Control and Optimization 56.4 (2018), pp. 2959-2976.

[2] P. Barrieu and N. El Karoui. "Pricing, hedging, and designing derivatives with risk measures." In: Indifference pricing: Theory and applications. Princeton, NJ: Princeton University Press, 2009, pp. 77-146. 
[3] V. I. Bogachev. Measure theory. Vol. 1. Springer Science \& Business Media, 2007.

[4] F. H. Clarke. "On the inverse function theorem". In: Pacific Journal of Mathematics 64.1 (1976), pp. 97-102.

[5] F. Delarue. "On the existence and uniqueness of solutions to FBSDEs in a non-degenerate case". In: Stochastic Process. Appl. 99.2 (2002), pp. 209-286.

[6] A. Fromm. "The method of decoupling fields generalized to higher spatial derivatives". In: arXiv preprint arXiv:1804.10970 (2018).

[7] A. Fromm. "Theory and applications of decoupling fields for forward-backward stochastic differential equations". PhD thesis. Humboldt-Universität zu Berlin, Mathematisch-Naturwissenschaftliche Fakultät II, 2015.

[8] A. Fromm and P. Imkeller. Existence, Uniqueness and Regularity of Decoupling Fields to Multidimensional Fully Coupled FBSDEs. 2013. arXiv: 1310.0499 [math.PR].

[9] Y. Hu and S. Peng. "Solution of forward-backward stochastic differential equations". In: Probability Theory and Related Fields 103.2 (1995), pp. 273-283.

[10] J. Ma, P. Protter, and J. M. Yong. "Solving forward-backward stochastic differential equations explicitly - a four step scheme". In: Probab. Theory Related Fields 98.3 (1994), pp. 339-359.

[11] J. Ma, Z. Wu, D. Zhang, and J. Zhang. "On well-posedness of forward-backward SDEs - a unified approach". In: Ann. Appl. Probab. 25.4 (2015), pp. 21682214 .

[12] J. Ma and J. Yong. Forward-backward stochastic differential equations and their applications. Vol. 1702. Lecture Notes in Mathematics. Springer-Verlag, Berlin, 1999.

[13] J. McNamara, A. Houston, and E. J. Collins. "Optimality models in behavioral biology". In: SIAM Review 43.3 (2001), pp. 413-466.

[14] E. Pardoux and S. Tang. "Forward-backward stochastic differential equations and quasilinear parabolic PDEs". In: Probab. Theory Related Fields 114.2 (1999), pp. 123-150.

[15] S. Peng and Z. Wu. "Fully coupled forward-backward stochastic differential equations and applications to optimal control". In: SIAM J. Control Optim. 37.3 (1999), pp. 825-843.

[16] J. Wendt. "The Maximum Principle and Controlled Diffusion". Master's thesis. Friedrich-Schiller-Universität Jena, 2019. URL: https : // doi .org/10 . 22032/dbt. 39400.

[17] J. Yong. "Finding adapted solutions of forward-backward stochastic differential equations: method of continuation". In: Probability Theory and Related Fields 107.4 (1997), pp. 537-572. 